\title{
DYNAMIC PLANNING OF EXPERIMENTS FOR THE OPTIMISATION OF MANAGERIAL SCHEDULING
}

\author{
Tomáš Macák ${ }^{1}$, Jan Hron ${ }^{1}$ \\ ${ }^{1}$ Department of Managemt, Faculty of Economics and Management, Czech University of Life Sciences in Prague, \\ Kamycka 129, 16521 Prague 6, Czech Republic
}

\begin{abstract}
MACÁK TOMÁŠ, HRON JAN. 2016. Dynamic Planning of Experiments for the Optimisation of Managerial Scheduling. Acta Universitatis Agriculturae et Silviculturde Mendelianae Brunensis, 64(6): 20472051.

Time management has a crucial role in organizations and also in our personal lives. Managerial scheduling is an important tool for the time management, especially It can serve as a tool for the first phase, of time management - namely for effective planning. This paper focusses on finding the best possible setting for determining significant the best layout for activities according to the criteria of urgency and importance using a modified steepest ascent method, which can be referred as dynamic scheduling. This term indicates the nature of the method; wherein the experimental design space is changed to look for the best conditions for adjustment factors influencing a managerial process. Existing methods for layout optimization mentioned in the literature and conventionally implemented in practice have only shown local optima.
\end{abstract}

Keywords: dynamic planning, steepest ascent, experimental space, managerial scheduling

\section{INTRODUCTION}

The objective (of most industrial experiments) is to set the levels of design factors that will optimize the yield. This area of production statistics is mentioned as response surface methodology (RSM), it was described by Wilson and Box. Most RSM applications involve sequential progress. There are three actions that are carried out during the use of the RSM, which are the following: A screening experiment has a goal to reduce many potentially significant factors to relatively few potentially important factors that influence the yield. These type of experiments to be run more efficiently in the next phases, and fewer runs are required; Using knowledge from the first step to moving through the experimental area in an attempt to approach the local optimum. The most general method is known as steepest ascent; Optimization based on the progress, where a small region close to the optimum has been identified using the second step; a model is then estimated that approximates a real response function.
This model is typically a second-order model used to approximate the curvature near the optimum, and this can be used to determine optimal conditions for the design factors (Montgomery, 2005). In split-plot designs, a particular type of restricted randomisation occurs throughout the experiment. A simple factorial experiment can lead in a splitplot type of design because of the way in which the experiment was executed. In many industrial experiments, three situations often arise, as follows:

Some of the factors of interest can be hard to modify while the rest of factors is easy to vary. Therefore the treatment combinations for the experiment which is run should be determined by the ordering of these hard to modify factors. Experimental units can be processed together as a batch for one or more of the factors in a particular treatment combination; Experimental units should be treated individually, one right after the other.

It means the same treatment combination without resetting the factor settings for the following treatment combination. A structure of the steepest ascent progress can complicate all three steps 
of an RSM problem. Recently, many researchers have considered this issue. Lucas and $\mathrm{Ju}$ (1992) provided a simulation study to investigate the use of split-plot designs in industrial experiments. Their results confirmed that steepest ascent designs produce increased precision for the subplot factors while sacrificing precision for the whole plot elements. Box (1996) explained that completely randomized experiments are often impractical in industry; in contrast, he indicated that split-plot experiments are often extremely efficient and easier to run. To illustrate the difference in the analysis, Lucas and Hazel (1997) ran the known paperhelicopter experiment as a CRD and as a split plot. Letsinger, Myers and Lentner (1996) introduced randomization designs (BRD), which are designs that have two randomisations, making them similar to split-plot designs. Vining, Kowalski, and Montgomery (2004) proposed central composite designs that were specifically designed for splitplot experiments. They also provided a general proof showing that for certain design conditions, the ordinary least squares coefficient estimates are equivalent to the generalized least squares coefficient estimates for second-order models. Kowalski, Vining, Montgomery and Borror (2004) modified the proposed central composite designs of Vining, Kowalski and Montgomery (2004) to model both the process mean and variances within a splitplot structure. Moreover, Bisgaard and Steinberg (1997) focussed on a design algorithm to obtain D-optimal split-plot designs. They showed that the design matrices for the D-optimal split-plot designs and D-optimal CRDs are typically different; moreover, they clarified that split-plot experiments are often more efficient than CRDs Huang, Chen and Voelkel (1998) and Bingham and Sitter (1999a) discussed minimum-aberration (MA) designs for steepest ascent experiments; in these works, both the whole-plot and subplot factors had two levels. The researchers provided methods for determining the MA designs and provided tables for various combinations of whole-plot and subplot factors. Some design issues with two-level fractionalfactorial split-plot experiments, including where to split and where to fractionate, were presented in Bingham and Sitter (2001).

Moreover, a theoretical justification for these types of split-plot designs was described in Bingham and Sitter (1999b). Schoen (1999) proposed a method for manipulating the division of contracts over the different error strata of two-level experiments with nested errors. Also, Goos and Vandebroek (2001) proposed an exchange algorithm to obtain D-optimal split-plot designs. They showed that the design matrices for the D-optimal split-plot designs and D-optimal CRDs are typically different and that split-plot experiments are often more efficient than CRDs. Kowalski (2002) considered split-plot experiments in the context of robust parameter design. He constructed 24-run designs in two ways, namely by using the properties of a balanced incomplete block design and by semi-folding a 16-run design. The main objective of the present paper is to address managerial scheduling optimization using the steepest ascent method when the experiment is conducted as a split plot.

\section{METHODOLOGY AND DATA}

\section{The Steepest Ascent Method}

The steepest ascent method involves moving through the experimental region along a path that yields increases in the response. After a first-order model has been fit, the regression coefficients $\beta j$ from the model are used to determine the coordinates along the path. The movement along the path of steepest ascent is proportional to the magnitude of the regression coefficient, with the direction based on the sign of the coefficient (Montgomery, 2005). Assuming that the first-order model is fitted, then

$y=\beta_{0}+\beta_{1} x_{1}+\beta_{2} x_{2}+\ldots+\beta_{k} x_{k}$

The path of steepest ascent moves a distance $r$ away from the design centre in the direction of the maximum response with the spherical constraint .

The maximisation procedure of the response function uses Lagrange multipliers. Then

(2) $Q\left(x_{1}, x_{2}, \ldots, x_{k}\right)=\sum_{j=1}^{k} \beta_{j} x_{j}-\lambda\left(\sum_{(j=1)}^{k} x_{j}^{2}-r^{2}\right)$,

where $\lambda$ is the Lagrange multiplier. The maximisation requires taking the partial derivatives of $Q$ with respect to each $x j$, as well as with respect to $\lambda$. Setting these partial derivatives equal to 0 and solving yields $x_{j}=\beta_{j} / 2 \lambda$ (for $j=1,2, \ldots, k$ ), which gives the coordinate of $x_{i}$ on the path of steepest ascent. See Myers and Montgomery (1995) for more details.

\section{Dynamic planning benefits over existing (conventional) approaches (described in the literature)}

Dynamic planning approach is suitable for the assumption that the research is experimenting with a process or production system (perhaps a new economic plan or process). Because initial approximation of operational states of the system is not often close from the true optimum, the investigation goal should be to quickly approach to the general surroundings of the total optimum using trial progress.

Therefore the aim of the effective planning is not to changeable settings that provide us an optimal response but to search for a new operating area in which the investigation process is usually refined from the economic standpoint. In this case, the economic benefit (of using this dynamic planning approach compared with conventional) is equal to $191-168=23$ thousand CZK. This 
represents the percentage difference equal to 13.7 of the gap between traditional and dynamic scheduling techniques.

\section{Dynamic planning using the Steepest Ascent}

The procedure is shown in the following example. Monitored response $\mathrm{Y}$ depends on two factors, where $\boldsymbol{x}_{\mathbf{1}}$ is the importance of the task (measured in thousands of monetary income in (ZK) and $\boldsymbol{x}_{2}$ is the urgency of the task (measured in the remaining days to the deadline). We will complete a design with three central points for a full schedule (see Table I).

\section{RESULTS}

The following figure is used to visualise the process of coding and decoding of two the factors.

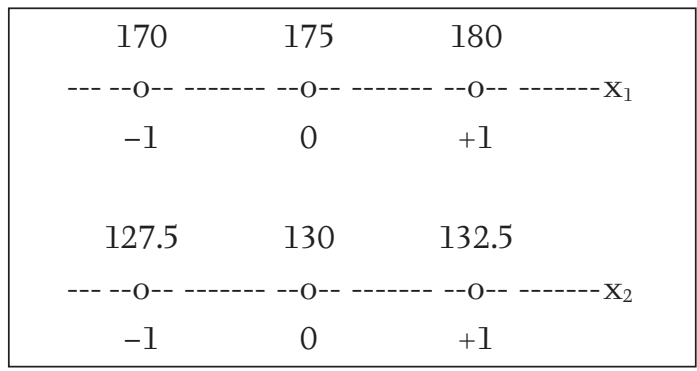

1: The conversion of coded values to operating values.

The coding and decoding equations are as follows: For $x_{1}$,

(3) $\xi_{1}=\frac{\left(x_{1}-175000\right)}{5}$ then $x_{1}=5 \xi_{1}+175$.

For $x_{2}$,

(4) $\xi_{2}=\frac{\left(x_{2}-130\right)}{2.5}$ then $x_{2}=2.5 \xi_{2}+130$.

Effects of a factor can be calculated as the average value of response for upper level of the factor minus the average value of the response factor for the lower level of the factor. Let the letters (1), a, b and ab represent all four combinations of the levels of the two factors ( $\mathrm{A}$ and $\mathrm{B}$ ), and let $\mathrm{n}$ be the number of replications of this experiment. Then, for the estimation, the effect of the ' $\mathrm{A}$ ' factor is valid, as

(5) $\operatorname{ef}(A)=\bar{y}_{A^{+}}-\bar{y}_{A^{-}}=\frac{a+a b}{2 n}-\frac{b+(1)}{2 n}=\frac{1}{2 n}[a+a b-b-(1)]$.

For our case calculation, the effect of the factors is

(6) $\operatorname{ef}\left(x_{1}\right)=\frac{1}{2}(-154300+160300-164600+168000)=78800$,

(7) $\operatorname{tf}\left(x_{2}\right)=\frac{1}{2}(164600+168000-160300-154300)=9000$.
The mean values of the response $\mathrm{Y}$ in the first plan also include the response of the central points:

(8) $\bar{Y}=\frac{1}{7}\left(\begin{array}{c}154300+160300+164600+168000+ \\ +160300+164300+162300\end{array}\right)=162014$.

The linear model includes the following equation: (9) $Y=162014+\frac{78800}{2} x_{1}+\frac{9000}{2} x_{2}=162014+39400 x_{1}+4500 x_{2}$.

The direction of the movement is determined by a vector with coordinates that are factor coefficients $\mathrm{x}_{1}$ and $\mathrm{x}_{2}$ in the linear model:

(10) $(39400,4500)$.

We can adjust the coordinates to simplify further calculations as

(11) $\frac{39400}{39400}=1 ; \frac{39400}{4500}=8.76$.

Multiples of these coordinates are coded values of factors. These points will be used in exploratory trials.

Because the goal is to maximise $\mathrm{Y}$ as the new centre point with the coded values'coordinates (3; 26.28), the new centre in real coordinates is then

(12) $\begin{aligned} x_{1} & =5 \xi_{1}+175=5 \times 3+175=190, \\ x_{2} & =2.5 \xi_{2}+130=2.5 \times 5.73+130=144.3 \cong 145 .\end{aligned}$

We set a wider spread of values around a new centre point, setting upper and lower limits to obtain more space for analysis, as follows:

\begin{tabular}{|c|c|c|}
\hline 180 & 190 & 200 \\
\hline --- --O-- & $--\mathrm{O}--$ & $--\mathrm{O}^{--}-------\mathrm{X}_{1}$ \\
\hline-1 & 0 & +1 \\
\hline 140 & 145 & 150 \\
\hline --- --O-- & $--\mathrm{O}--$ & $--\mathrm{O}^{--}------\mathrm{X}_{2}$ \\
\hline-1 & 0 & +1 \\
\hline
\end{tabular}

2: The second conversion of coded values to operating values.

The coding and decoding equations are as follows: For $\mathrm{x}_{1}$,

(13) $\xi_{1}=\frac{\left(x_{1}-190\right)}{10}$ then $x_{1}=10 \xi_{1}+190$.

For $\mathrm{x}_{2}$ :

(14) $\xi_{1}=\frac{\left(x_{2}-145\right)}{5}$ then $x_{2}=5 \xi_{2}+145$.

The second plan is shown in Table III. 
For our second plan calculation, the effects of the factors are as follows:

(15) $(-178000+184000-191000+177000)=-4000$,

(16) $\operatorname{ef}\left(x_{2}\right)=\frac{1}{2}(191000+177400-184000+178000)=3000$.
The mean values of the response $Y$ in the first plan also include the response of the central points:

(17) $19100+177000+189000+186000)=184167$.

In the second plan, the linear model has the following equation:

(18) $Y=184167-2000 x_{1}+1500 x_{2}$.

I: Monitored Response Y Depends on Two Factors, $x_{1}$ and $x_{2}$

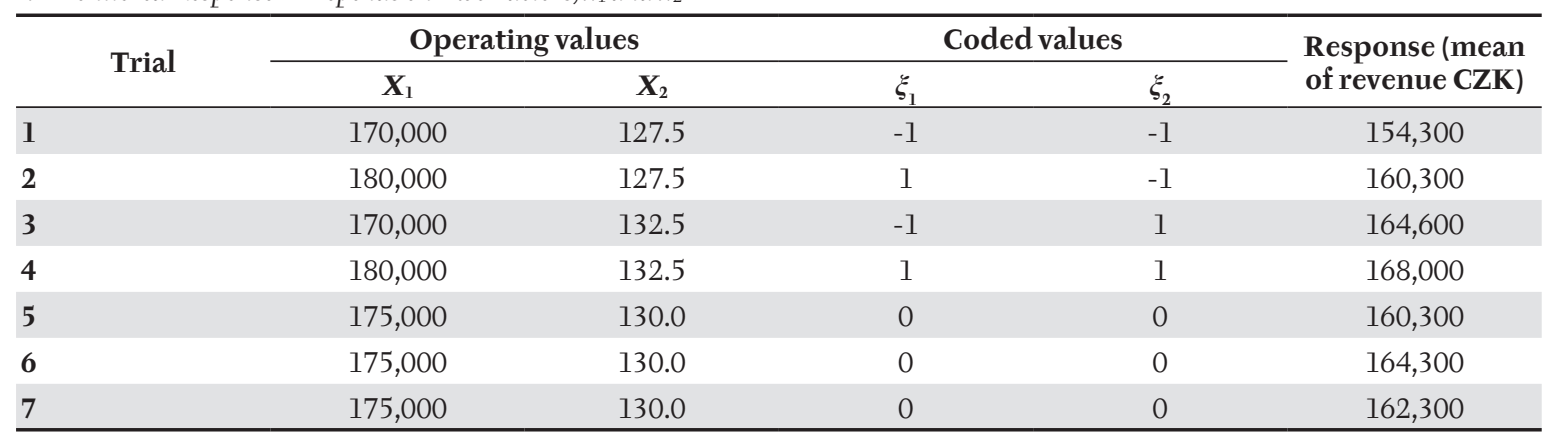

II: Coordinates for the Exploratory Experiment Results

\begin{tabular}{lccc}
\hline Run & $\mathbf{X}_{\mathbf{1}}$ coordinate & $\mathbf{X}_{\mathbf{2}}$ coordinate & $\begin{array}{c}\text { Response Y } \\
\text { (mean of revenue CZK) }\end{array}$ \\
\hline $\mathbf{1}$ & 1 & 8.76 & 174,300 \\
$\mathbf{2}$ & 2 & $17.52=2 \times 8.76$ & 179,800 \\
$\mathbf{3}$ & 3 & $26.28=3 \times 8.76$ & 186,500 \\
$\mathbf{4}$ & 4 & $35.04=4 \times 8.76$ & 167,400 \\
\hline $\mathbf{5}$ & 5 & $43.8=5 \times 8.76$ & 158,700 \\
\hline
\end{tabular}

III: The Second Plan by Dynamic planning

\begin{tabular}{|c|c|c|c|c|c|}
\hline \multirow{2}{*}{ Trial } & \multicolumn{2}{|c|}{ Operating value } & \multicolumn{2}{|c|}{ Coded value } & \multirow{2}{*}{ Response Y (CZK) } \\
\hline & $\mathbf{X}_{1}$ & $\mathbf{X}_{2}$ & $\xi 1$ & $\xi 2$ & \\
\hline 1 & 180 & 140 & -1 & -1 & 178,000 \\
\hline 3 & 180 & 150 & -1 & 1 & 191,000 \\
\hline 4 & 200 & 150 & 1 & 1 & 177,000 \\
\hline 6 & 190 & 145 & 0 & 0 & 186,000 \\
\hline
\end{tabular}

Because the variance of general response is greater than the square of the difference between the mean response and the response of the central points, the procedure is over. 


\section{DISCUSSION AND CONCLUSIONS}

The aim of the paper was to introduce a dynamic method for management activities planning. The method used two variables - urgency and time priority. Dynamic scheduling can be here mark used because the optimal allocation of resources does not take place in only one feasible area, but the method allows to search other areas of potential solutions. The advantage of the method is based on its application because it can be applied without specialized software and also permits to perform more complex solution than an optimization using convection methods (e.g. linear programming or factor analysis).

Often, due to cost or time constraints, it is not feasible to completely randomize an experiment, and this is especially the case in managerial scheduling. In such a situation, some factors are hard to change. Many tests in the industry are RSM studies, where the objective is to identify the optimal conditions for the design elements. One important step in an RSM study is moving through the region using the path of steepest ascent toward an area where the optimum lies. It would be virtually impossible to run one operate at a time in a split-plot setting because of the randomization limitation. Thus, we have introduced a simplified procedure based on the steepest ascent principle without using Lagrange multipliers; this method can be used to handle managerial scheduling without complicated calculation.

\section{Acknowledgements}

The authors would like to thank the editor and an anonymous reviewer for their insightful comments on an earlier version of this paper. We would like to express our gratitude to Dr Petr Juza, general manager of the Agrofarma Krasna, for enabling execution of the managerial scheduling experiment.

\section{REFERENCES}

BINGHAM, D. and SITTER, R. S. 2001. Design Issues in Fractional Factorial Split-Plot Experiments. Journal of Quality Technology, 33: 1-15.

BISGAARD, S. 2000. The Design and Analysis of Split Plot Experiment. Journal of Quality Technology, 32:39-56.

BOX, G. E. P. 1996. Split-Plot Experiments. Quality Engineering, 8: 515-520.

BOX, G. E. P. and JONES, S. 1992. Split-Plot Designs for Robust Product Experimentation. Journal of Applied Statistics, 19:3-26.

GOOS, P. and VANDEBROEK, M. 2001. Optimal Split-Plot Designs. Journal of Quality Technology, 33: 436-450.

HUANG, P., CHEN, D., and VOELKEL, J. O. 1998. Minimum- Aberration Two-Level Split-Plot Designs. Technometrics, 40: 314-326.

KOWALSKI, S. M., VINING, G. G., MONTGOMERY, D. C.; and BORROR, C. M. 2004. Modifying a Central Composite Design to Model the Process Mean and Variance When There Are Hard-toChange Factors. Journal of the Royal Statistical Society: Series C (Applied Statistics), 55: 615-630.

LETSINGER, J. D., MYERS, R. H.; and LENTNER, M. 1996. Response Surface Methods for BiRandomization Structures. Journal of Quality Technology, 28: 381-397.
LUCAS, J. M. and HAZEL, M. C. 1997. Running Experiments With Multiple Error Terms: How an Experiment Is Run Is Important. In: ASQC Technical Conference Transactions. Milwaukee, WI: American Society for Quality Control, 283-296.

MYERS, R. H., MONTGOMERY, D. C.; VINING, G. G., BORROR, C. M., and KOWALSKI, S. M. 2004. Response Surface Methodology: A Retrospective and Literature Survey. Journal of Quality Technology: ASQC Technical Conference Transactions, 36: 53-77.

VINING, G. G., KOWALSKI, S. M., and MONTGOMERY, D. C. 2005. Response surface designs within a split-plot structure. Journal of Quality Technology, 37(2): 115-129. 